\title{
Autorinnen und Autoren dieses Heftes
}

Tobias Böhmelt

Margit Bussmann

Christopher Daase

Benjamin Herborth

Julian Junk

Martin Nonhoff

Diana Panke

Ulrich H. Pilster
Dr., Wissenschaftlicher Mitarbeiter der Professur für Internationale Beziehungen an der ETH Zürich, Haldeneggsteig 4, 8092 Zürich, Schweiz,

E-Mail: tobias.boehmelt@ir.gess.ethz.ch

Dr., Professorin für Internationale Politik und Regionalstudien am Institut für Politik- und Kommunikationswissenschaft der Ernst Moritz Arndt Universität Greifswald, Baderstraße 6/7, 17489 Greifswald,

E-Mail: margit.bussmann@uni-greifswald.de

Dr., Professor für Internationale Organisation am Institut für Politikwissenschaft/Fachbereich 03 Gesellschaftswissenschaften im Rahmen des Exzellenzclusters »Die Herausbildung normativer Ordnungen « an der Goethe-Universität Frankfurt am Main, Senckenberganlage 31, 60325 Frankfurt a. M.,

E-Mail: daase@normativeorders.net

M.A., Assistant Professor of Political Science an der University of Groningen, Oude Kijk in't Jatstraat 5/9, 9700 AS Groningen, Niederlande,

E-Mail: herborth@soz.uni-frankfurt.de

Dipl.-Verw.Wiss., Wissenschaftlicher Mitarbeiter am Institut für Politikwissenschaft/Fachbereich 03 Gesellschaftswissenschaften und im Exzellenzcluster »Die Herausbildung normativer Ordnungen « an der Goethe-Universität Frankfurt am Main, Senckenberganlage 31, 60325 Frankfurt a. M., E-Mail: julian.junk@normativeorders.net

Dr., Wissenschaftlicher Mitarbeiter am Zentrum für Sozialpolitik sowie am SFB $597 »$ Staatlichkeit im Wandel« der Universität Bremen, Mary-Somerville-Str. 5, 28359 Bremen, E-Mail: mnonhoff@zes.uni-bremen.de

Dr., Associate Professor an der School of Politics and International Relations des University College Dublin, Dublin 4, Irland,

E-Mail: diana.panke@ucd.ie

Dr., Analyst am Allied Command Operations (ACO) der NATO in Belgien und Visiting Fellow am Department of Government der University of Essex, Wivenhoe Park, Colchester CO4 3SQ, Großbritannien, E-Mail: ulrich.pilster@gmail.com 
Andreas Vasilache

Dr., Professor als Juniorprofessor für Sozialwissenschaftliche Europaforschung an der Universität Bielefeld und Direktor des Centre for German and European Studies (CGES/ZDES) an der Universität Bielefeld und der Staatlichen Universität St. Petersburg, Postfach 100131, 33501 Bielefeld, E-Mail: andreas.vasilache@uni-bielefeld.de 